\title{
Atomic and electronic structure in collapsed carbon nanotubes evidenced by scanning tunneling microscopy
}

\author{
Cristina E. Giusca, Yann Tison, and S. Ravi P. Silva* \\ Nano-Electronics Centre, Advanced Technology Institute, University of Surrey, Guildford, Surrey GU2 7XH, United Kingdom
}

(Received 21 December 2006; revised manuscript received 2 April 2007; published 24 July 2007)

\begin{abstract}
The electronic behavior of a radially collapsed armchair carbon nanotube encountered by scanning tunneling microscopy experiments is presented in a study that probes the electronic changes directly associated with the atomically resolved structural perturbations. The finite density of states obtained through scanning tunneling spectroscopy at the Fermi energy when the interspacing of the flattened faces does not allow for bond formation suggests that the electronic properties are slightly modified under mild radial deformations and provides a striking verification of previous theoretical predictions and discussions. The study clearly illustrates the challenges to be faced in the contacting of future nanowires, predicted to be the active component in integrated circuits.
\end{abstract}

DOI: 10.1103/PhysRevB.76.035429

PACS number(s): 73.22. $-\mathrm{f}$

\section{INTRODUCTION}

Scanning tunneling microscopy (STM) experiments can be used to examine the properties of carbon nanotubes, which are predicted by atomistic calculations, and to verify the electronic properties expected by measurement of the local density of states in these one-dimensional structures. However, these materials are not always perfect hexagonal lattice cylinders and they can develop defects during their growth, processing, or characterization. Structural deformations have often been observed in STM and atomic force microscopy (AFM) experiments on carbon nanotubes revealing bent and twisted tubes. ${ }^{1,2}$ Some of these defects are also associated with a rearrangement of atoms and bonds which will impact on the band structure, affecting thus the electronic properties of the tube. AFM experiments and molecular mechanics simulations ${ }^{1}$ have shown that the van der Waals forces between nanotubes and the substrate on which they are placed can lead to significant deformations of their structure as nanotubes tend to follow the topography of the substrate in order to maximize their adhesion energy. In this process, buckles or kinks can develop in the nanotube structure, when the strain accumulated exceeds a structural limit. ${ }^{3}$ STM and scanning tunneling spectroscopy (STS) measurements on kinklike structures have shown that they seamlessly connect metallic and semiconducting parts of the same tube, ${ }^{4}$ in agreement with theoretical predictions that two nanotubes with different chiralities can be connected together by a pentagon-heptagon pair. ${ }^{5}$

A different type of deformation, predicted theoretically and evidenced experimentally by transmission electron microscopy and AFM, ${ }^{6,7}$ refers to the carbon nanotubes' susceptibility of collapsing radially into a flattened tube with bulbs on either edge, in a geometry that balances the curvature energy with the interlayer interaction. ${ }^{8}$ In this case, the large radial deformations are stabilized by the intersheet attraction between opposing sides of the wall. It was shown that collapse is favored in large tubes: as the tube circumference increases, the excess curvature energy in the bulbs becomes constant, while the energetic advantage of the intersheet attraction increases linearly.., 10
Depending on the nanotube diameter, a map of stability was obtained by atomistic simulations for the deformation of an $(n, n)$ armchair single-walled carbon nanotube (SWNT), showing the possible regimes for partially and fully collapsed nanotubes and revealing the influence of the commensurability of the constituent layers, which induces further mechanical deformations beyond the flattening, such as twisting and warping. ${ }^{11}$

In view of using carbon nanotubes for fabricating electronic devices, it is important to understand to what extent these deformations affect their electronic properties. Theoretical studies have also been performed in order to identify the correlation between the structural perturbations and the change in the electronic properties, and some of these studies predict remarkable changes. For example, the electronic properties of a squashed armchair nanotube determined using tight-binding molecular dynamics calculations demonstrate a metal to semiconductor transition upon squashing the nanotube. ${ }^{12,13}$ Similar deformations increase the gap in small-gap zigzag tubes, and gap closure can occur due to enhanced $\sigma^{*}-\pi^{*}$ hybridization effects. ${ }^{14}$

We present here observations of a collapsed carbon nanotube encountered by STM experiments and confirm changes in the electronic properties due to structural perturbations, in a study that correlates the atomically resolved structure to the electronic behavior of the deformed tubes as studied by STS.

\section{EXPERIMENTAL DETAILS}

For STM experiments, a suspension of carbon nanotubes was prepared by sonication in 1,2-dicholoroethane for approximately 15 min and then drop casting on Au substrates prepared as described in Ref. 15. The sample was left in the fast entry lock of the system prior to introducing it into the STM chamber and pumped overnight to allow for the specimen to outgas. The instrument used for the STM studies is a commercial Omicron STM/SEM HC, combining STM with scanning electron microscopy. Prior to STM experiments, scanner calibration was carried out on highly oriented pyrolitic graphite (HOPG), which also helped with assessing the quality of the tip by rendering the atomic resolution. STM 

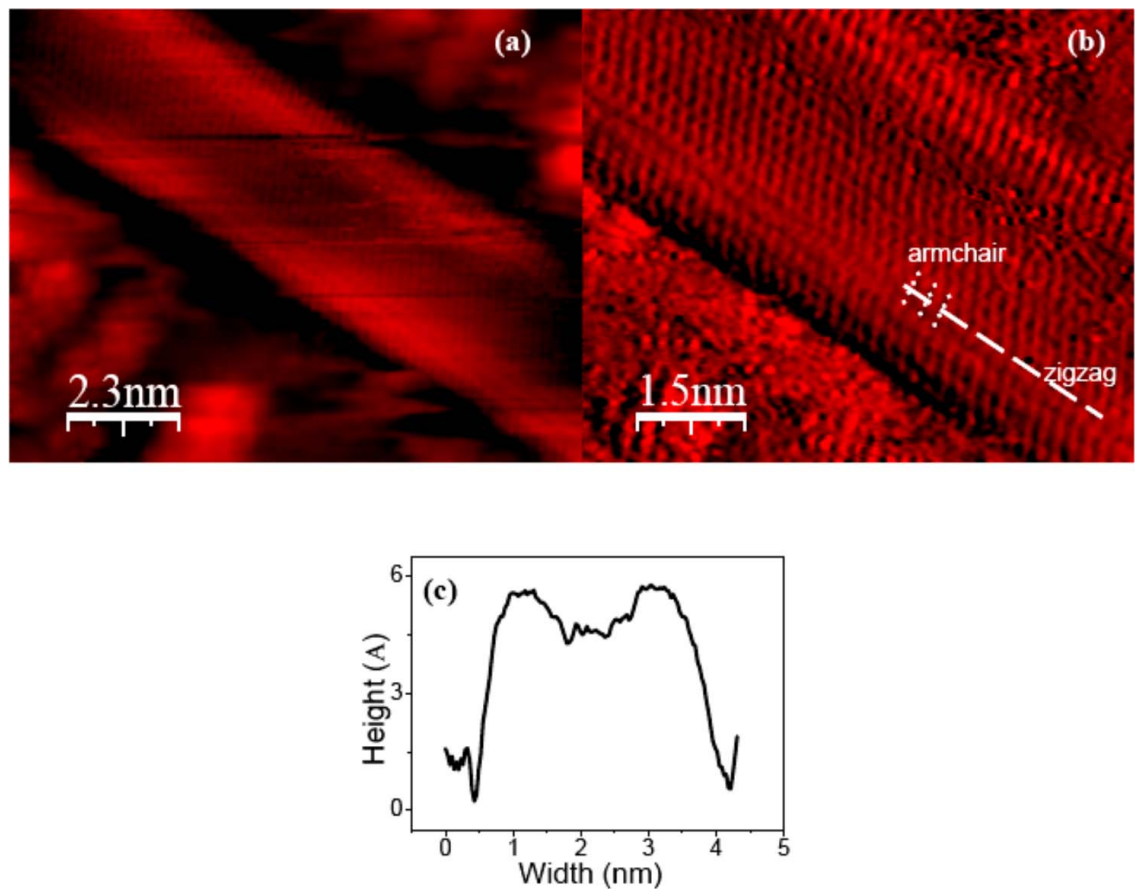

tips were electrochemically etched $\mathrm{W}$ wire prepared immediately before introducing them into the UHV chamber and their cleanliness was checked against HOPG. $I-V$ curves recorded periodically during experiment on clean regions of the $\mathrm{Au}$ substrate displaying metallic behavior also provide confirmation of a suitably clean STM tip. All measurements were carried out at room temperature in constant current mode. Typical scanning conditions used with this sample were $0.1 \mathrm{nA}$ for the tunneling current and a positive sample bias of $0.1 \mathrm{~V}$. The STS measurements were performed by interrupting the scanning and the feedback at preset locations and recording the tunneling current variations during the sample bias ramp (usually between -2 and $+2 \mathrm{~V}$ ). STM images were processed using WSxM software. ${ }^{34}$

\section{RESULTS AND DISCUSSION}

The STM experiments revealed a few separate occurrences of nanotubes clearly showing radial deformation of tubes, flattened to ribbonlike structures, indicating the formation of collapsed configurations, as illustrated by Figs. 1(a) and 1(b). The carbon nanotube (CNT) in Fig. 1(a) displays two distinct sections, also seen when examined at higher magnification in Fig. 1(b) a flat ribbonlike shape along its length in the central region and a bulblike shape at each side of the edges. As also indicated by a cross-sectional profile along a line perpendicular to the nanotube axis [Fig. 1(c)], this particular shape suggests a centrally collapsed nanotube.

The atomically resolved image allows for the determination of the tube chiral angle. The chiral angle and the tube diameter are linked to the chiral indices $(n, m)$ of the tube which determine whether a SWNT will be a metal or a semiconductor. ${ }^{16,17}$ The chirality determination in the case of carbon nanotubes is complicated by the finite size of the STM tip and the circular shape of the tube. The geometry of
FIG. 1. (Color online) STM images of the collapsed tube. (a) Atomically resolved STM image showing the centrally collapsed carbon nanotube and (b) a zoom-in image of the nanotube shown in (a). The dark spots on the surface of the nanotube wall represent the centers of the hexagons of the nanotube lattice. Derivative image is shown as it reveals enhanced borders and more contrast, highlighting the distinct features of the tube. (c) Typical height profile obtained by averaging over 15 cross-sectional profiles across the tube. the tip apex results in convolution effects, producing an apparent broadening of the nanotube, whereas the circular shape of the tube, combined with the tendency of the tunneling current to follow the shortest path, induces distortions in the STM image of the nanotube lattice, making it to appear stretched in the direction perpendicular to the tube. ${ }^{18-20}$

Generally, for an accurate diameter determination, models for deconvoluting the tip contribution are employed, ${ }^{21}$ which might not yield a very precise determination in the present case due to the noncircular shape of the nanotube and to the unknown geometry of the STM tip.

For the present case, a good estimation of the tube diameter for the collapsed case would be given by total-energy calculations which take into account the interlayer attraction between the opposing walls. Our estimation for the diameter of the tube in its uncollapsed state uses standard geometry, assuming that the cross section of the collapsed tube can be represented by the shape shown in Fig. 2(a), where the two bulbs at the edges modeled to have circular cross sections are joined by a flat region of length $l$. The diameter of the tube in its uncollapsed state was determined by averaging between two extreme cases, as illustrated in Figs. 2(b) and 2(c). One case corresponds to the situation wherein the opposing internal faces of the tube come into contact $(d=0)$, as shown in Fig. 2(b), whereas for the other case, the tube is only flattened without an inflexion in the central region [Fig. 2(c)].

Using the notations introduced in Fig. 2(b), the perimeter of the collapsed tube can be written to a gross approximation as twice the perimeter of two semicircles joined by the length $l$ :

$$
P_{\text {collapsed }}=2\left(\pi r_{1}+\pi r_{2}+l\right) \quad \text { with } l=L-2 r_{1}-2 r_{2} .
$$

Because the perimeter of the tube should remain constant in either the collapsed or the uncollapsed state, we can estimate the diameter $D$ of the uncollapsed (cylindrical) tube from 

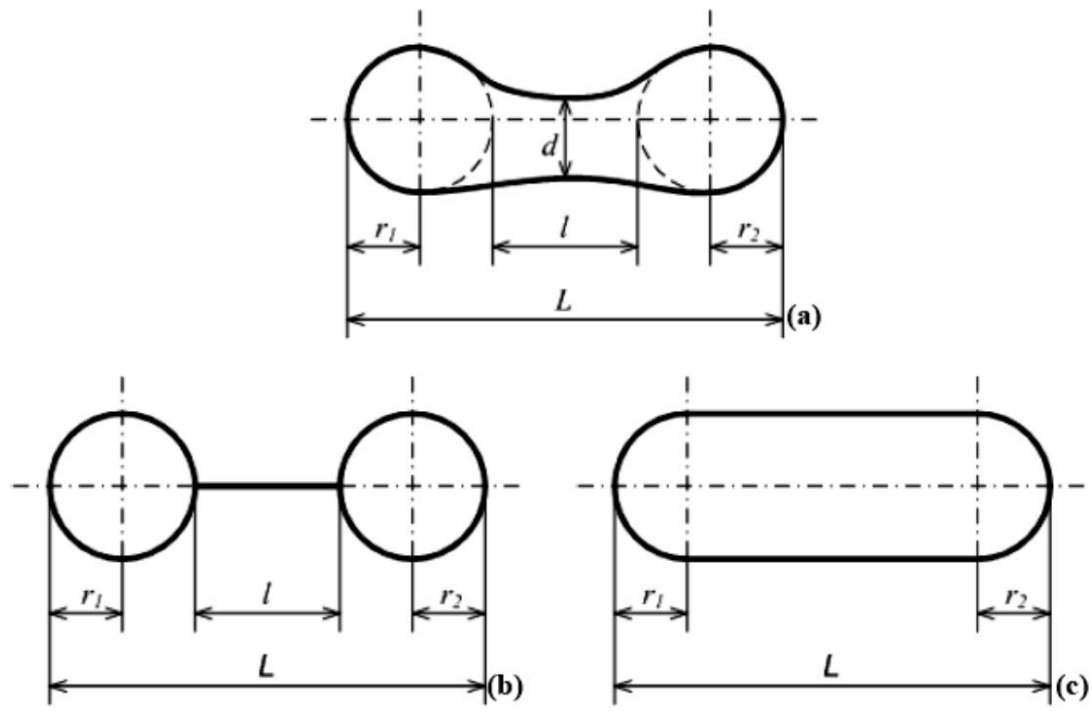

$P_{\text {collapsed }}=P_{\text {uncollapsed }}=\pi D, \quad D=2 / \pi\left[L+(\pi-2)\left(r_{1}+r_{2}\right)\right]$.

With $L, r_{1}$, and $r_{2}$ derived from the experimental line profiles as the one shown in Fig. 1(c) a value of $2.98 \mathrm{~nm}$ is obtained for the diameter of the undeformed tube. The values for $L(3.47 \mathrm{~nm}), r_{1}(0.5 \mathrm{~nm})$, and $r_{2}(0.5 \mathrm{~nm})$ were obtained by averaging measured data points from 15 line profiles across the tube.

For the case presented in Fig. 2(c), corresponding to a lower attraction between the walls than in the previous case, but still greater than the curvature energy, the diameter can be estimated from

$$
\begin{aligned}
P_{\text {collapsed }}= & \left(\pi r_{1}+\pi r_{2}+2 l\right)=P_{\text {uncollapsed }}=\pi D \quad \text { with } l=L \\
& -r_{1}-r_{2} .
\end{aligned}
$$

The experimental values for $L, r_{1}$, and $r_{2}$ yield a $2.61 \mathrm{~nm}$ value for the diameter $D$, which by averaging with the value obtained in the previous case $(2.98 \mathrm{~nm})$ gives an estimated value of $\sim 2.79 \mathrm{~nm}$ for the tube diameter $D$ in its uncollapsed state.

The estimated value of the uncollapsed tube diameter can give an indication of the number of constituent layers for the tube under investigation. When measuring the tube heights in relation to the surface from the experimental cross-sectional profiles, we estimate a value of $\sim 0.40 \mathrm{~nm}$ for the distance $d$ between the opposing walls. This interspacing distance can only be consistent with a SWNT, as it is of the order of the typical interlayer value for graphite $(0.34 \mathrm{~nm})$. A doublewalled nanotube would yield a value equal to three times the graphite interlayer spacing, i.e., $\sim 1.02 \mathrm{~nm}$ for the distance between the two flat faces of the outer tube, which clearly is not the case here and therefore we conclude that it is a SWNT.

Having determined the diameter, we can now estimate the chirality of the SWNT based on the atomically resolved image. In order to avoid contributions coming from the sides of the tubes, the chiral angle is best measured on the structure visible on top of the tube, as sides can be distorted by the effects stated above. ${ }^{22}$ For the present case, the structure at the top of the nanotube is reasonably flat on a sufficiently large scale as to ensure that these effects do not affect the correct chiral angle, which gives a value of $30^{\circ}$. Careful analysis of the $\mathrm{C}$ hexagons on the bulbs reveals that their alignment is such that the $\mathrm{C}-\mathrm{C}$ bonds are oriented perpendicular to the tube axis, giving a chiral angle of $30^{\circ}$, characteristic to the unique armchair-type structure. Closer inspection also reveals fluctuations within $\left(2^{\circ}-3^{\circ}\right)$ of the angle between the armchair and the zigzag direction from $90^{\circ}$ which is generally the value for an armchair tube. These fluctuations could be the result of a twist of the nanotube, responsible for modifying the angle between the zigzag and armchair direction, ${ }^{2}$ as highlighted in Fig. 3 (inset), showing the distortion of the hexagon as a result of a twist. The $\mathrm{C}-\mathrm{C}$

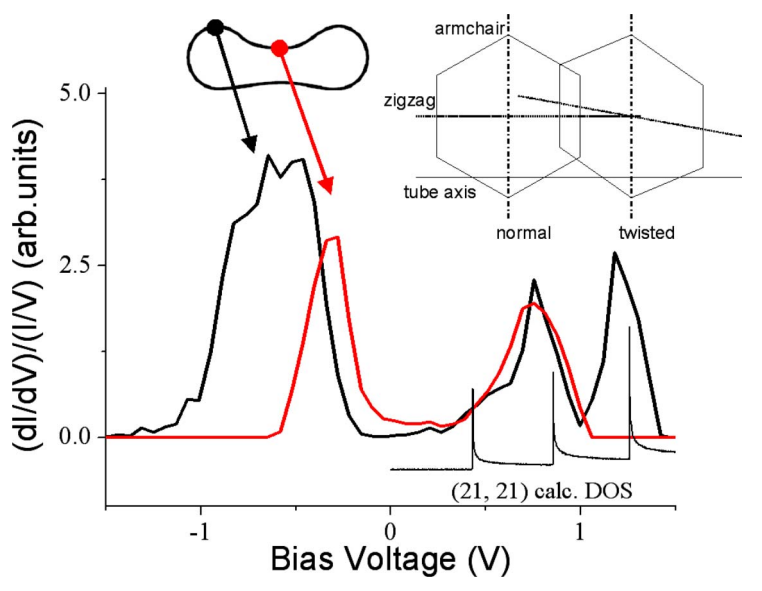

FIG. 3. (Color online) Tunneling spectra recorded by STS on the collapsed tube. The normalized differential conductance, which is a measure of the DOS, was computed for the SWNT shown in Fig. 1. The spectra were recorded on the flat central region and on the bulbs at the edges of the nanotube as indicated by arrows. The calculated $\operatorname{DOS}^{24}$ showing only the conduction band (due to symmetry reasons) for a $(21,21)$ SWNT is included for comparison and shifted vertically for clarity. Also included is a schematic representation for the normal and twisted cases, illustrating the angle variation between the armchair and zigzag directions observed on the collapsed tube (top right). 
bond alignment is such that the zigzag direction with respect to the armchair fluctuates from $90^{\circ}$, while the armchair direction remains on average perpendicular to the tube axis, which is not the case for a chiral tube.

As recently shown, ${ }^{11}$ the commensurability between layers can be responsible for the formation of various deformation structures in collapsed nanotubes. Atomistic simulations ${ }^{11}$ reveal that different stacking between the commensurate and the incommensurate state induces the existence of a rotational shift between the two adhering layers in the energy minimization process, which is responsible for introducing a twist or a warping (a " " shape) structure. The observed twist of the SWNT could thus be attributed to a rotation of the two flat opposing layers toward a more energetically stable structure, possibly toward achieving the perfect $A B$ stacking, which was suggested to be a more stable formation than the $A A$ stacking. ${ }^{10,11}$ In addition to being the result of the nanotube following the substrate features, visible distortions of the $\mathrm{C}$ hexagons and apparent height variations along the tube could also support a rotation of the flat layers in the attempt of achieving a more energetically favorable structure.

A $(21,21)$ pair of chiral indices can be assigned to the collapsed SWNT based on the values determined for the chiral angle and diameter. We estimate an accuracy of $0.1 \mathrm{~nm}$ and $\sim 1^{\circ}$ for the measurement of the diameter and chiral angle, respectively. Such a tube is expected to exhibit metallic behavior. It is well known that minor variations of the diameter and wrapping angle can drastically affect the electronic properties of the CNTs. Here, however, due to the special value of the chiral angle for this tube, the range of possible diameters determined from the experimental crosssectional profiles will not change its metallic behavior. As estimated above for the two extreme cases, the diameter can vary between 2.61 and $2.98 \mathrm{~nm}$, corresponding thus to four possible values for the chiral indices $n=m=19,20,21$, and 22 , all making the tube metallic. On the stability map of the most energetically favorable $(n, n)$ armchair structures ${ }^{11}$ all these possible chiralities belong to the collapse metastable region, suggesting that there might exist other more favorable energetically structures, such as twisted or warped SWNT.

To correlate the atomic structure of this tube to its electronic properties, STS spectra have been recorded along the tube and the normalized differential conductance calculated for each curve to yield the density of states (DOS) ${ }^{23}$ Spectra were recorded at three different locations along the tube (corresponding to points close to the beginning, middle, and end of the scan) on the flat central region and also at three other locations on each bulb at the sides, showing reproducibility and repeatability along the tube. The results are displayed in Fig. 3.

Typical tunneling spectra recorded on the SWNT (Fig. 3) show the peaks corresponding to the Van Hove singularities at the onsets of the one-dimensional energy bands of the carbon nanotube. The peaks are asymmetric and broadened by the hybridization with the Au substrate, resembling well the overall shape of the calculated $\operatorname{DOS}^{24}$ but observed to have shifted by $\sim 0.1 \mathrm{eV}$ to the left, toward the valence band, indicating doping by the substrate. The flat region at the left and right sides in the spectra taken on the collapsed region, and so the absence of the second Van Hove singularity above $1 \mathrm{eV}$, is due to the preamplifier saturation and can also be considered as evidence for the better conductivity we observe for the collapsed part.

The tunneling spectra can be used to determine the nanotube diameter, and as for a metallic nanotube theory predicts the following relation for the separation between the energy band edges: $E_{g}^{m}=6 \gamma_{0} a_{\mathrm{C}-\mathrm{C}} / d{ }^{25}$ where $a_{\mathrm{C}-\mathrm{C}}$ is the distance between two neighboring $\mathrm{C}$ atoms $(0.142 \mathrm{~nm})$ and $d$ the diameter of the nanotube. $\gamma_{0}$ is the value of the nearest neighbor overlap integral, ranging from 2.4 to $2.9 \mathrm{eV} .{ }^{19,26,27}$ The range of diameters so obtained for the currently investigated nanotube is from 2.5 to $3 \mathrm{~nm}$ for a $0.8 \mathrm{eV}$ separation, assuming that the positions of the Van Hove singularities are not affected by the radial deformation.

The most prominent feature, however, is the difference in DOS at the Fermi level. The tunneling spectra recorded on the central flat region show a finite DOS at the Fermi level, which is consistent with the metallic nature, while on the bulbs at either side of the tube an opening of a gap is observed with a zero DOS, indicative of a semiconducting nature. The zero DOS is consistent for all curves recorded on the bulbs, but in contrast with the predicted metallic behavior for an armchair tube.

An $(n, n)$ armchair SWNT has $n$ mirror planes containing the tube axis, with two energy levels characterized by $\pi$ and $\pi^{*}$, which are even and odd under mirror symmetry operations. ${ }^{14}$ These levels are degenerate at the Fermi level, resulting in metallic behavior. It was shown that when $(n, n)$ tubes are elongated and compressed in the axial direction, while maintaining a circular cross section, the mirror symmetry is preserved and the metallic conduction remains. However, radial deformation can break the mirror symmetry, depending on the radial direction of the deformation, ${ }^{8,12,13}$ and when the tube collapses, the normally radially separated areas of the nanotube are brought into close proximity and so the low-energy electronic properties of the CNT altered. It was predicted by theory ${ }^{12}$ that upon transverse collapse of CNTs, metallic $(n, n)$ tubes become semiconductors and narrow-gap $(3 n, 0)$ tubes can become metallic. Any gap in a collapsed $(n, n)$ nanotube arises purely from the coupling of the interior faces of the flat region. The coupling mixes states with $k$ related by reflection across the tube axis and results in quadratic energy dispersion, exhibiting a gap, which was calculated to range from 0.04 to $0.2 \mathrm{eV}$ at room temperature and zero pressure for a $(20,20)$ tube.

Another study of the metal to semiconductor transition in a squashed armchair nanotube involving tight-binding molecular dynamics ${ }^{13}$ shows that the metal to semiconductor transition has to be driven by a combined effect of bond formation and mirror symmetry breaking. To open a gap, the atomic orbitals of the two flattened faces of the squashed tube without mirror symmetry have to overlap with each other and form new bonds. It follows that one condition for obtaining a gap requires the distance between the two opposing walls to allow for the bond formation between equivalent $\mathrm{C}$ atoms. The spacing we obtain between the two opposing layers is of the order of $\sim 0.40 \mathrm{~nm}$, greater than $0.26 \mathrm{~nm}$ 
which is the length of an interplanar C-C bond. It is worth stating that the possibility for the interlayer spacing to be close to $0.26 \mathrm{~nm}$ is not completely excluded as the interlayer spacing was obtained using height information extracted from multiple cross-sectional profiles and the apparent height profile is known to be highly dependent on the imaging conditions (bias voltage, etc.), on the adsorption distance of the nanotube above the substrate, and different tunneling distances on the nanotube and on the substrate. However, the bonding alone cannot open a gap if the mirror symmetry is preserved, and conversely, the mirror symmetry breaking alone only induces a variation in the conductance, without opening a gap. ${ }^{13}$ The mirror symmetry breaking is in direct relation with the existence of the two equivalent sublattices ( $A$ and $B$ ) of graphene. In reference to the $A$ and $B$ sublattices, if the axis along which the tube is squashed passes through two atoms of the same sublattice, the squashed tube will break the mirror symmetry about that axis but it will be maintained if the axis passes through atoms of different sublattices..$^{13}$

In other words, $A A$ stacking between the flat opposing walls will break the mirror symmetry, and if the walls are close enough so that $A$ atoms bond with $A$ atoms, a gap will be formed upon squashing. For $A B$ stacking, the mirror symmetry is preserved and the $\pi$ and $\pi^{*}$ bands cross without the gap opening, regardless of whether a bond exists between the two flattened faces. The exact stacking order for the flat region of our tube is difficult to determine from the existing STM images as some of the $\mathrm{C}$ atoms appear brighter than their neighbors, indicating thus the atoms having a $\mathrm{C}$ atom directly underneath and the $A B$ stacking, while other regions display uniform brightness for all six atoms of the hexagons, consistent with $A A$ stacking. ${ }^{28}$ The $A B$ stacking which does not allow for the mirror symmetry breaking adds to the bonding absence due to the observed large interlayer spacing, making observing a gap in the flat region unlikely. Mixed stacking could be related to the degree of commensurability of the two flat opposing walls of the nanotube and could also indicate an interlayer adjustment toward the more favorable structure, such as translation or rotation of one layer relative to the other and, a rotation could result in introducing the observed twist.

The twist would be consistent with the gap opening in the DOS observed on the bulbs, as a localized twist is a source of electron backscattering and a uniformly twisted SWNT opens a gap at the Fermi energy, in a metallic tube. ${ }^{29}$ Based on the determined value of the chiral angle which we believe is not affected by the tip effect distortions, on the $\mathrm{C}-\mathrm{C}$ bond orientation and the mixed stacking we observe that the $2^{\circ}-3^{\circ}$ fluctuations should correspond to a twist and not to a chirality in the nanotube. Note that the slight variation toward lower angles of the hexagon rows along the flat ribbon with respect to the tube axis from the left to the right of the tube could also account for a small twist. For an untwisted tube, all hexagon rows should align parallel to each other along the tube. We estimate the difference in angle between the hexagon rows at the right and left of the tube to be $\sim 3^{\circ}$. This value coincides with the observed variation from $90^{\circ}$ of the angle between armchair and zigzag directions, giving further confirmation for the existence of the twist. As expected, a twist would also alter the bond distance between $\mathrm{C}$ atoms within the hexagons, a detail confirmed by the STM images, showing evidence for elongated and compressed bonds relative to the known value for graphite $(0.14 \mathrm{~nm})$. This also contributes to the increase in the accumulated internal stress, which can alter the band structure due to possible rehybridization effects analogous to the gap opening in small diameter nanotubes ${ }^{30}$ or induce changes to the $s p^{2}$ bonding framework as in systems such as fullerene polymer $\left(\mathrm{C}_{60}\right)_{x}$ and fullerene adducts. These systems contain several $s p^{3}$ hybridized carbon atoms on the conjugated network of buckminsterfullerene, supposed to have carbons in an overall $s p^{2}$ hybridized state, and were found to be better described by $s p^{3.5}$ and $s p^{4.1}$ hybridization, respectively. ${ }^{31}$ Effects of stressinduced band structure modification have also been observed experimentally in highly compressive amorphous carbon thin films, where a decrease of an interplane $s p^{2}$ cluster distance reduction of about $15 \%$ was found as a result of highly localized strain, ${ }^{32}$ in agreement with the previous work of Umemoto et al. on foamlike systems based on rigidly interconnected segments of graphite. ${ }^{33}$

Pseudogaps have been observed for metallic zigzag tubes $^{30}$ and for armchair tubes packed within a rope. For the latter case, the gap opening was attributed to interactions with other tubes in the rope allowing for the $\pi$ and $\pi^{*}$ bands to mix. For the present case, the nanotube is not part of a bundle, and individual armchair tubes isolated on the substrate were found not to display a pseudogap, ${ }^{30}$ so the possibility of a pseudogap can be excluded.

Hertel et al. ${ }^{1}$ have used continuum mechanics and molecular mechanics simulations combined with AFM measurements to show that defects or steps on the substrate can induce substantial distortions in adsorbed nanotubes due to their strong adhesion to the substrate. Using the relation they obtain for the binding energy dependence on the diameter, we estimate a van der Waals binding energy of $\sim 0.2 \mathrm{eV} / \AA$ for a tube of $2.8 \mathrm{~nm}$ diameter. Due to the large binding energies involved, Hertel et al. also find that the forces between the nanotube and features of the substrate such as steps or other defects can be extremely high, and they estimate a force of $\sim 35 \mathrm{nN}$ for a tube of $10 \mathrm{~nm}$ diameter, with a $1 \mathrm{eV} / \AA ̊$ in binding energy, ${ }^{1}$ which would result in a force of $\sim 9 \mathrm{nN}$ for our case, presumably responsible for inducing the collapse we observe.

\section{CONCLUSIONS}

An armchair SWNT in a collapsed configuration was observed by STM and the experimental observations on the electronic behavior substantiate the validity of previous theoretical studies on radially deformed tubes. Atomically resolved images are consistent with mixed stacking suggesting the influence of the lattice commensurability as a likely reason for shifting the opposing layers relative to each other toward a minimum-energy state, in accordance to earlier studies. As a result, the twist-induced deformation alters the metallicity of the armchair structure and a gap is observed on the bulbs of the collapsed tube. Finite density of states at the Fermi energy found on the central ribbon region when the 
interlayer spacing does not allow for the bond formation between equivalent $\mathrm{C}$ atoms constitutes the first experimental test of earlier theoretical calculations. Our experiments show that the electronic properties of armchair nanotubes are slightly disturbed under mild radial deformations provided further deformations are not induced and might find applications for nanotube device fabrication allowing for fine and local tuning of their electronic properties. The results clearly indicate the delicate nature of the carbon nanotubes and, in general, the significant change in electronic properties of nanowires when subject to local stresses and/or pressure.
This has serious implications in the design of nanowire integrated circuits predicted to replace planar complementary metal-oxide semiconductor devices and also shows that care needs to be taken when probing the electronic properties of such structures.

\section{ACKNOWLEDGMENT}

The authors with to acknowledge the financial support afforded by the EPSRC, UK.
*Corresponding author; s.silva@surrey.ac.uk

${ }^{1}$ T. Hertel, R. E. Walkup, and Ph. Avouris, Phys. Rev. B 58, 13870 (1998).

${ }^{2}$ W. Clauss, D. J. Bergeron, and A. T. Johnson, Phys. Rev. B 58, R4266 (1998).

${ }^{3}$ A. Rochefort, P. Avouris, F. Lesage, and D. R. Salahub, Phys. Rev. B 60, 13824 (1999).

${ }^{4}$ M. Ouyang, J. L. Huang, C. L. Cheung, and C. M. Lieber, Science 291, 97 (2001).

${ }^{5} \mathrm{Ph}$. Lambin, A. Fonseca, J. P. Vigneron, J. B. Nagy, and A. A. Lucas, Chem. Phys. Lett. 245, 85 (1995).

${ }^{6}$ M.-F. Yu, M. J. Dyer, J. Chen, D. Qian, W. K. Liu, and R. S. Ruoff, Phys. Rev. B 64, 241403(R) (2001).

${ }^{7}$ M.-F. Yu, T. Kowalewski, and R. S. Ruoff, Phys. Rev. Lett. 86, 87 (2001).

${ }^{8}$ N. G. Chopra, L. X. Benedict, V. H. Crespi, M. L. Cohen, S. G. Louie, and A. Zettl, Nature (London) 377, 135 (1995).

${ }^{9}$ L. X. Benedict, N. G. Chopra, M. L. Cohen, A. Zettl, S. G. Louie, and V. H. Crespi, Chem. Phys. Lett. 286, 490 (1998).

${ }^{10}$ A. N. Kolmogorov and V. H. Crespi, Phys. Rev. Lett. 85, 4727 (2000)

${ }^{11}$ B. Liu, M. Yu, and Y. Huang, Phys. Rev. B 70, 161402(R) (2004).

${ }^{12}$ P. E. Lammert, P. Zhang, and V. H. Crespi, Phys. Rev. Lett. 84, 2453 (2000).

${ }^{13}$ J.-Q. Lu, J. Wu, W. Duan, F. Liu, B.-F. Zhu, and B.-L. Gu, Phys. Rev. Lett. 90, 156601 (2003).

${ }^{14}$ C.-J. Park, Y.-H. Kim, and K. J. Chang, Phys. Rev. B 60, 10656 (1999).

${ }^{15}$ M. Hegner, P. Wagner, and G. Semenza, Surf. Sci. 291, 39 (1993).

${ }^{16}$ J. W. G. Wildöer, L. C. Venema, A. G. Rinzler, R. E. Smalley, and C. Dekker, Nature (London) 391, 59 (1998).

${ }^{17}$ T. W. Odom, J. L. Huang, P. Kim, and C. M. Lieber, Nature (London) 391, 62 (1998).

${ }^{18}$ P. Lambin, G. I. Mark, V. Meunier, and L. P. Biró, Int. J. Quantum Chem. 95, 493 (2003).

${ }^{19}$ L. C. Venema, V. Meunier, P. Lambin, and C. Dekker, Phys. Rev. B 61, 2991 (2000).

${ }^{20}$ J. Vidal, R. Mosseri, and B. Doucot, Phys. Rev. Lett. 81, 5888 (1998).

${ }^{21}$ P. Kim, T. W. Odom, J. L. Huang, and C. M. Lieber, Carbon 38, 1741 (2000).

${ }^{22}$ T. W. Odom, J. L. Huang, P. Kim, M. Ouyang, and C. M. Lieber, J. Mater. Res. 13, 2380 (1998).

${ }^{23}$ J. A. Stroscio, R. M. Feenstra, and A. P. Fein, Phys. Rev. Lett. 57, 2579 (1986).

${ }^{24}$ http://www.photon.t.u-tokyo.ac.jp/ maruyama/nanotube.html

${ }^{25}$ J.-C. Charlier and Ph. Lambin, Phys. Rev. B 57, R15037 (1998).

${ }^{26}$ M. S. Dresselhaus, G. Dresselhaus, and P. C. Eklund, Science of Fullerenes and Nanotubes (Academic, San Diego, CA, 1996).

${ }^{27}$ C. T. White and J. W. Mintmire, Nature (London) 394, 29 (1998).

${ }^{28}$ A. Hassanien, A. Mrzel, M. Tokumoto, and D. Tomanek, Appl. Phys. Lett. 79, 25 (2001).

${ }^{29}$ C. L. Kane and E. J. Mele, Phys. Rev. Lett. 78, 1932 (1997).

${ }^{30}$ M. Ouyang, J. L. Huang, C. L. Cheung, and C. M. Lieber, Science 292, 702 (2001).

${ }^{31}$ P. R. Surjan, J. Mol. Struct.: THEOCHEM 338, 215 (1995).

${ }^{32}$ C. H. Poa, R. G. Lacerda, D. C. Cox, F. C. Marques, and S. R. P. Silva, Appl. Phys. Lett. 81, 853 (2002).

${ }^{33}$ K. Umemoto, S. Saito, S. Berber, and D. Tomanek, Phys. Rev. B 64, 193409 (2001).

${ }^{34} \mathrm{http}: / /$ www.nanotec.es 